\title{
Behavioral and EEG changes after 14 days of perceptual deprivation'
}

\author{
John P. Zubek \\ UNIVERSITY OF MANITOBA, CANADA
}

\begin{abstract}
A progressive decrease in frequencies in the alpha range was observed during 14 days exposure to unpatterned light and white noise. Large individual differences, however, were present. Measures of CFF, depth perception, and size constancy were not affected and hallucinatory-like phenomena were rare. However, in several cases, long-lasting motivational losses were observed.
\end{abstract}

\section{Problem}

In an exploratory study, using $3 \mathrm{Ss}$, we observed a decrease in mean occipital lobe frequency during a 14-day exposure to perceptual deprivation. Furthermore, follow-up records taken a week after the termination of isolation still revealed some signs of EEG abnormality. Hallucinatory phenomena and postisolation disturbances of the perceptual environment were rare (Zubek et al., 1963). Since this preliminary study, an additional $7 \mathrm{Ss}$ have been isolated. The present paper summarizes our results on these 10, 14-day Ss.

\section{Method}

A group of 15 male Ss were placed individually in an isolation chamber for a prescribed period of two weeks. Of these, $5 \mathrm{Ss}$ failed to endure the full duration; all terminated isolation within the first four days. The Ss wore translucent goggles which permitted light but excluded all pattern vision. Each Salso wore heavy gloves and a set of earmuffs through which white noise, somewhat above the threshold of hearing, was constantly presented. He was not permitted to sing, hum, or engage in any other vocal activity. No restrictions, however, were placed on his motor activity. EEGs were taken before isolation and then during isolation at intervals of 7,10 , and 14 days. Follow-up records were also taken at $1,2,7$, and 10 days after termination of isolation. The mean occipital lobe frequency was then determined by the method of Engel et al., (1944). This involved counting the number of waves occurring in each of 200 , 1-sec. samples of artifact-free occipital lobe tracings. In addition to EEGs, measures of depth perception (Howard-Dohlman apparatus), CFF, and size constancy were taken before and after the 14-day isolation period. A group of 15 controls also received these three tests, 14 days apart.

\section{Results and Discussion}

Table 1 shows a progressive decrease in mean occipital lobe frequency with time in isolation. Furthermore, the mean decrease during the second week (1.11 cps) is approximately twice as great as that during the first (0.52 cps). Unfortunately, this difference only borders on statistical significance. Nevertheless, the trend is unmistakable. Table 1 also indicates a progressive increase in mean frequency following termination of isolation. However, even after 10 days there are still

Table 1. Mean Occipital Lobe Frequencies at Various Intervals During and After 14 Days of Perceptual Deprivation.

\begin{tabular}{|c|c|c|c|c|c|c|c|c|}
\hline \multirow{2}{*}{ Subject } & \multicolumn{4}{|c|}{ During Deprivation } & \multicolumn{4}{|c|}{ After Deprivation } \\
\hline & Day 0 & Day 7 & Day 10 & Day 14 & Day 1 & Day 2 & Day 7 & Day 10 \\
\hline 1 & 10.10 & 9.16 & 8.60 & 7.15 & 7.89 & 8.62 & 9.57 & --- \\
\hline 2 & 13.03 & 12.65 & 11.40 & 10.44 & 11.04 & 11.34 & 12.50 & --- \\
\hline 3 & 11.56 & --- & 10.14 & 8.00 & 10.21 & --- & 11.01 & -- \\
\hline 4 & 11.27 & 10.13 & 9.87 & 9.08 & 9.61 & 9.83 & 10.14 & 10.68 \\
\hline 5 & 9.67 & 9.27 & 8.75 & 8.39 & 8.57 & 8.97 & 9.18 & 9.46 \\
\hline 6 & 11.51 & 11.04 & 10.93 & 9.96 & 10.35 & 10.54 & 10.36 & 10.94 \\
\hline 7 & 10.66 & 10.06 & 10.02 & 9.72 & 10.01 & 10.30 & 10.45 & 10.50 \\
\hline 8 & 10.92 & 10.65 & 10.63 & 10.66 & 10.75 & 10.67 & 10.67 & 10.65 \\
\hline 9 & 10.65 & 10.70 & 10.56 & 10.16 & 9.91 & 10.04 & 10.38 & 10.61 \\
\hline 10 & 10.46 & 10.50 & 10.42 & 9.90 & 10.11 & 10.32 & 10.47 & 10.44 \\
\hline Mean & 10.98 & 10.46 & 10.13 & 9.35 & 9.84 & 10.07 & 10.47 & 10.47 \\
\hline
\end{tabular}


some indications of EEG abnormality. Although these physiological changes, in general, are quite large they would undoubtedly have been evengreater if restrictions had been placed on Ss motor activity (Zubek \& Wilgosh, 1963).

Perhaps the most important finding is the existence of large individual differences. As the table indicates, the mean decrease in frequency, after the 14-day period, ranges from 3.56 to $0.26 \mathrm{cps}$. This suggests, therefore, that certain individuals can withstand conditions of reduced sensory input much better than others. Similar results have also been observed in prison situations. Hinkle (1961), for example, has reported that "under prison isolation, as this has been carried out by Russian and Eastern European state police, most prisoners developed symptoms of disorganization within three to six weeks; but some have been known to endure this for many months, and some have succumbed within days." Large differences also exist in the capacity to withstand the effects of sleep deprivation. Some people can endure $100 \mathrm{hr}$. with their functions largely intact whereas others become disorganized and ineffective after only $48 \mathrm{hr}$. of sleep deprivation (Hinkle, 1961).

Depth perception, CFF, and size constancy were not impaired, a finding in agreement with our earlier 7-day experiments. This suggests that certain basic perceptual processes are not affected by even prolonged periods of reduced sensory input. Finally, since 14-day Ss are rare, some mention will be made of their subjective reactions. As far as hallucinatory phenomena are concerned, they were almost totally lacking. The few that occurred were exclusively of the simple Type I variety. Furthermore, upon emerging from isolation, none of the Ss reported any gross disturbances of the perceptual environment of the type that were observed at McGill.
There were, however, some motivational losses characterized by inability to study or to engage in a variety of activities. These persisted from less than a day to 8 days (mean $=31 / 2$ days). As might be expected, some relation existed between the magnitude of the EEG decrease and the duration of the motivational losses (Rho $=0.67)$. Finally, one volunteer (S4, Table 1) reported a strong craving to go back into isolation shortly after his release. Such a reaction has been observed in our laboratory only once before-after 10 days of darkness and silence. It may, therefore, only occur after very long periods of deprivation. It is interesting to note that some long-term prison inmates as well as Polar explorers and inhabitants have also reported a similar phenomenon-a strong desire to return to their former isolated environment.

\section{References}

ENGEL, G. L., ROMANO, J., FERRIS, E. B., WEBB, J. P., \& STEVENS, C. D. A simple method of determining frequency spectrums in the electroencephalogram. Arch. Neurol. Psychiat., 1944, 51, 134-146.

HINKLE, L. E. The physiological state of the interrogation subject as it affects brain function. In A. D. Biderman \& H. Zimmer (Eds.), The manipulation of human behavior. New York: Wiley, 1961. Pp. 19-50.

ZUBEK, J. P., \& WILGOSH, L. Prolonged immobilization of the body: changes in performance and in the electroencephalogram. Science, 1963, 140, 306-308.

ZUBEK, J. P., WELCH, G., \& SAUNDERS, M. G. EEG changes during and after 14 days of perceptual deprivation. Science, 1963, 139, 490-492.

\section{Note}

1. This study was supported by the Defence Research Board, Canada (project 9425-08). Grateful acknowledgement is made to Dr. M. G. Saunders, Winnipeg General Hospital, for the provision of EEG facilities. 